\title{
Psicometria e pósvenção ao suicídio: Revisão e bibliometria de pesquisas
}

\author{
Psychometrics and postvention: Review and research bibliometrics \\ Psicometría e intervención post-suicidio: Revisión e bibliometría
}

\author{
Alessandro Vieira dos Reis \\ ORCID: https://orcid.org/0000-0002-2581-492X \\ Universidade Federal de Santa Catarina, Brasil \\ E-mail: alessandrovr@gmail.com \\ Janete Knapik \\ ORCID: https://orcid.org/0000-0002-3449-1376 \\ Universidade Positivo, Brasil \\ E-mail: janete.knapik@up.edu.br
}

\begin{abstract}
Resumo
O suicídio é um problema multideterminado, tanto de ordem individual quanto de saúde pública. Por isso envolve diferentes procedimentos de prevenção e de tratamento dos enlutados. Nesse último quesito encontra-se o conceito de pósvenção, que consiste em estratégias de ajuda psicológica a familiares, amigos e conhecidos de suicidas para uma melhor elaboração do luto, evitando assim novos transtornos e complicações. A produção acadêmica sobre pósvenção ainda é escassa. Sobretudo no que diz respeito a procedimentos quantitativos, como os que fazem uso de questionários psicométricos, que objetivam medir construtos psicológicos. Diante de tal problemática, este artigo tem por objetivo levantar como a Psicometria vem sendo usada para fundamentar intervenções em pósvenção ao suicídio. Para realizar esse objetivo, foi empreendida uma revisão integrativa de publicações, seguida de análise bibliométrica dos artigos selecionados. Constam como principais resultados da pesquisa: a) o levantamento de publicações acadêmicas em Psicometria da pósvenção a nível mundial; b) análise dos construtos psicológicos que são focos de atenção; c) crítica do conceito de pósvenção à luz da psicometria utilizada.
\end{abstract}

Palavras-chave: Pósvenção; Suicídio; Psicometria; Avaliação psicológica.

\begin{abstract}
Suicide is a multi-determined problem, both individual and public health. Therefore, it involves different prevention and treatment procedures for the bereaved. In this last item is the concept of post-invention, which consists of psychological help strategies for family members, friends and acquaintances of suicidal people for a better elaboration of grief, thus avoiding new disorders and complications. Academic research on postvention is still scarce. Especially with regard to quantitative procedures, such as those that use psychometric questionnaires, which aim to measure psychological constructs involved. Faced with this problem, this research aims to raise how Psychometry has been used to support interventions in postvention. To accomplish this objective, a systematic review of publications was undertaken, followed by bibliometric analysis of the selected papers. The main results of the research are: a) a selection of the most relevant surveys about Psychometrics in postvention around the world; $b$ ) the analysis of the psychological constructs that are the focus of attention; c) a criticism of the concept of postvention in light of the psychometrics.
\end{abstract} Keywords: Postvention; Suicide; Psychometry; Psychological assessment.

\section{Resumen}

El suicidio es un problema multidisciplinario, tanto individual como de salud pública. Por lo tanto, implica diferentes procedimientos de prevención y tratamiento para los afligidos. En este último ítem se encuentra el concepto de postinvención, que consiste en estrategias de ayuda psicológica a familiares, amigos y conocidos de personas suicidas para una mejor elaboración del duelo, evitando así nuevos trastornos y complicaciones. La producción académica sobre postventión es aún escasa. Especialmente en lo que respecta a los procedimientos cuantitativos, como los que utilizan cuestionarios psicométricos, que tienen como objetivo medir constructos psicológicos. Ante este problema, este artículo tiene como objetivo plantear cómo se ha utilizado la Psicometría para apoyar intervenciones en intervenciones postsuicidio. Para lograr este objetivo, se realizó una revisión integrativa de las publicaciones, seguida de un análisis bibliométrico de los artículos seleccionados. Los principales resultados de la investigación son: a) el relevamiento de publicaciones académicas en Psicometría postventión a nivel mundial; b) análisis de los constructos psicológicos que son el foco de atención; c) crítica al concepto de posventión a la luz de la psicometría utilizada. Palabras clave: Posvención; Suicidio; Psicometría; Evaluación psicológica. 


\section{Introdução}

O neologismo "pósvenção", derivado de "prevenção", data de 1968, tendo sido cunhado em uma conferência sobre saúde mental e suicídio na África do Sul para designar estratégias de intervenção que ocorrem após um caso de suicídio, visando o cuidado com os enlutados (New York State Psychiatric Institute, 2020). Os primeiros programas e protocolos de pósvenção surgiram nos anos 2000, na América do Norte. Desses destacam-se o da California State University (2006), nos EUA; e, no Canadá, o programa da Associação de Prevenção ao Suicídio de Quebec (AQPS, 2008).

Enquanto objeto de estudo científico, a pósvenção vem despertando interesse acadêmico no campo multidisciplinar da Saúde Mental. Sobretudo na Psiquiatria e na Psicologia, mas também na Assistência Social, Sociologia, Enfermagem, etc. Diante de tal problemática, esta pesquisa tem por objetivo investigar como a Psicometria, braço técnico-científico da Psicologia marcado por pesquisas quantitativas (Pasquali, 2009) vem fundamentando intervenções em pósvenção. Entende-se que o tratamento de risco de suicídio consiste numa questão delicada que exige máximos cuidados em termos avaliativos e deliberativos por parte dos profissionais envolvidos. O que justifica a busca de rigor e criteriosidade científica no que tange os instrumentos empregados.

Este estudo tem por objetivo levantar o uso de instrumentos psicométricos validados que façam parte de estratégias de pósvenção e analisar as mesmas à luz da Psicometria. Para tal foi empreendida uma revisão integrativa integrativa de publicações, seguida de análise bibliométrica dos resultados. O escopo deste estudo restringe-se em oferecer uma panorâmica da Psicometria aplicada à pósvenção, e não apontar os melhores procedimentos e instrumentos ou propor um programa de intervenção.

\section{Revisão de Literatura}

\subsection{Suicídio e luto}

Definível como o ato voluntário de tirar a própria vida, o suicídio começa a ser estudado cientificamente no século XIX, na Sociologia de Durkheim. O autor, em "O Suicídio", publicado em 1897 (Durkheim, 1982), teoriza que o fenômeno pode ser explicado por um estado de anomia do indivíduo. A anomia consistiria, segundo o autor, na ausência de regramento sadio na sociedade moderna, que ocasiona a perda do senso de identidade e de pertencimento (Nunes, 1998). O século XX viu surgir, nos campos da Psiquiatria e da Psicologia, outras teorias sobre o suicídio, como as baseadas em transtornos psiquiátricos. Destacase entre eles, segundo o DSM-V, a depressão e demais transtornos de humor como fatores de explicação do suicidio (American Psychiatric Association, 2014). Há um amplo acordo, contudo, que o ato de tirar a própria vida é um fenômeno multideterminado e de difícil compreensão.

O suicídio hoje é considerado um problema de saúde pública e a mortalidade e tem aumentado muito nos últimos anos (Kreuz, \& Antoniassi, 2020; Fukumitsu, 2014; Ibrahim, Che Din, Amit, Ghazali \& Mohd Safien, 2019). Estatísticas divulgadas pela Organização Mundial de Saúde revelaram que mais de 800.000 pessoas morreram por suicídio em 2012, sendo essa a segunda causa de morte mais prevalente de pessoas entre 15 e 29 anos no mundo (World Health Organization, 2016). Uma pessoa que atenta contra a própria vida rompe uma fronteira e escancara uma pergunta insistente no que concerne às motivações que o levaram a querer deixar de existir, aniquilar-se. Segundo Kreuz e Antoniassi (2020), para cada suicídio aproximadamente 100 pessoas são afetadas, com pouco ou nenhum suporte para lidar com a tragédia, e o luto por suicídio tem particularidades que podem dificultar o processo natural do luto. Sendo assim, as ações de intervenção após um suicídio são um importante aporte de apoio que abriga cada enlutado em sua dor particular e única, mas também reconhece a dor que é coletiva.

Pessoas enlutadas por suicídio estão especialmente vulneráveis e correm também o risco de morrer de suicídio. Por exemplo, Wainwright et al (2020) identificaram a importância de os pais enlutados não se sentirem sós, as dificuldades para se ter acesso a suporte psicológico de grupos e à informações sobre vias de apoio. Os enlutados carregam consequências para saúde mental como angústias e emoções negativas tais como culpa, vergonha, desamparo e raiva. Observa-se nessas pessoas o aumento 
nos índices de transtornos do humor, em especial a depressão, de ansiedade, de estresse pós-traumático, assim como dificuldades sociais como tentativas de achar culpados e um "bode expiatório" na família ou na comunidade, estigmatizações, evitação de famílias e amigos e dissoluções de casamentos (Jordan, 2017).

\subsection{Prevenção e pósvenção}

O trabalho de prevenção do suicídio, que consiste em identificar e abordar adequadamente os potenciais suicidas, enfrenta múltiplas dificuldades por conta do aspecto complexo e delicado de tratar o que compete ao fenômeno. Por conta disso, existem diversos modelos de prevenção ao suicídio. Nesse contexto de prevenção desponta o conceito de "pósvenção", que consiste em estratégias de suporte aos enlutados para uma melhor resolução da crise instaurada, bem como evitar novos casos de suicídio por contágio (Ramchand et al, 2015). Enquanto a prevenção tem, por definição, um caráter de monitoramento précrise, a pósvenção, por sua vez, consiste em estratégias para respoder à seguinte pergunta: “O que fazer com as pessoas que sobrevivem ao suicídio?". Em outras palavras, aos enlutados e demais afetados psicologicamente: todos os que experimentam forte crise decorrente da perda e correm riscos psicológicos e até físicos.

As buscas na internet pelo termo "postvention" apresentam uma tendência de crescimento, conforme exposto na Figura 1:

Figura 1 - Buscas por "postvention".

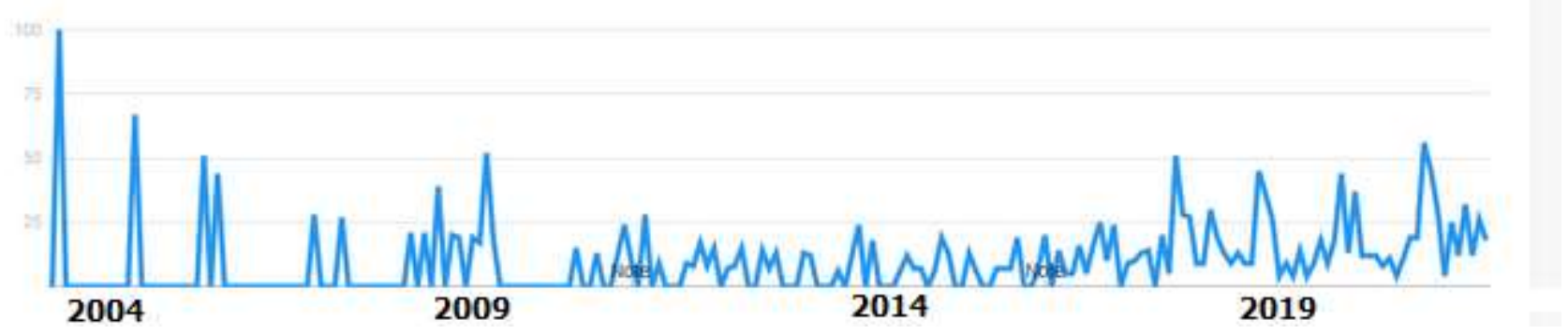

Fonte: Google Trends.

O interesse crescente na pósvenção, observado na Figura 1 sobretudo a partir da segunda metade da década de 2010, desperta a crítica quanto às evidências científicas no embasamento de tais intervenções. Evidências essas muitas vezes ainda apontadas como fracas ou inexistentes (Ramchand et al., 2015).

Grande parte do trabalho de pósvenção encontra-se na facilitação do luto. Isto é, na elaboração saudável de tal processo intensamente doloroso. O luto pode ser saudável, no sentido de natural ou esperado diante de trágicas perdas. Mesmo no luto sem complicações, alguns indivíduos respondem a perda com sintomas característicos de um episódio de depressão maior como tristeza associados a sintomas como insônia, perda de apetite e peso e a duração do luto pode variar dependendo de diferenças culturais (APA, 2014). Já o luto que ultrapassa os limites do esperado desencadeia ou agrava transtornos mentais pré-existentes. Esse luto patológico seria marcado pelos seguintes sintomas: durar mais de um ano, perda da identidade, mais marcado por sentimento de perda do que pela incapacidade de sentir felicidade (Michel e Freitas, 2019).

A elaboração do luto em trabalhos de pósvenção é especialmente dificultada porque o luto por suicidas é um tabu. A perda de uma pessoa por suicídio costuma ser um assunto interditado, o que explica porque os enlutados e demais afetados não sabem como lidar com o processo.

Historicamente, o início das práticas em pósvenção se deu em instituições escolares, envolvendo adolescentes (Earbecher, Singer \& Poland, 2015). Estatisticamente, a maior parte dos “aglomerados de suicídios", isto é, suicídios por imitação, são formados por adolescentes, segmento da sociedade mais influenciável pelo contágio do suicídio (Andrienssem, 2019). No entanto, programas de pósvenção não ocorrem apenas em instituições escolares. Os mesmos também podem se dar 
em hospitais psiquiátricos (Olinda, Santos e Marques, 2020); e corporações militares (Santos, 2019). No ambiente militar dos EUA, por sua vez, observou-se o surgimento de um dos primeiros programas sistemáticos de pósvenção amparados por larga pesquisa (Ramchand et al, 2015). Tal programa consiste em quatro etapas: a) vigilância (o que incluiu casos suspeitos e autópsias psicológicas), b) prevenção (risco de 'clusters'), c) suporte ao luto (risco de fadiga e distress, uso de Cognitive Behavioral Therapy), d) respeito e honra ao falecido e entes queridos.

Já no que tange a divulgação do conceito de pósvenção no Brasil, uma das primeiras referências foi a publicação, em 2017, pelo Ministério da Saúde, de uma cartilha de prevenção ao suicídio que fala de pósvenção (Guimarães, Santos \& Lima, 2020). Constam como outras iniciativas de divulgação: publicações no portal do Instituto Vita Alere, na cidade de São Paulo (Vita Alere, 2018); uma matéria do Jornal da USP sobre pósvenção (Fukumitsu, 2018); o folder da Secretaria de Justiça do DF, datado de 2019 (Secretaria de Cidadania e Justiça do Distrito Federal, 2019) e o folder informativo do Conselho Regional de Psicologia do Distrito Federal (CRP 12, 2020).

Em termos de publicações acadêmicas que citam a pósvenção, observa-se que os mais antigos no Brasil datam de 2017:

- $\quad$ artigo no Psicologia.PT, sobre elaboração do luto pós-suicídio em ambiente familiar (Silva e Marinho, 2017);

- $\quad$ artigo publicado sobre a atuação do profissional de Enfermagem em casos de pósvenção ao suicídio (Santos et al, 2017);

- $\quad$ artigo sobre como realizar pósvenção em Centros de Atendimento Psicossociais (Müller, Pereira e Zanon, 2017);

- trabalho de conclusão de curso em Psicologia sobre sobreviventes do suicídio (Barbosa, 2017);

- trabalho de conclusão de curso em Psicologia, sobre intervenções psicológicas em pósvenção (Knapik, Nogueira \& Ostrowski, 2019);

- trabalho de conclusão de curso em Psicologia sobre cuidados com enlutados pós-suicídio (Betti, 2020).

Os itens da lista acima foram resultados não aproveitados, por filtros seletivos empregados, na revisão integrativa (ver seção 2 deste artigo).

\subsection{Psicometria nos estudos sobre suicídio}

A Avaliação Psicológica consiste em um campo da Psicologia regulamentado pela Lei Federal nº 4119 (Presidência da República, 1962), que aborda “aspectos cognitivos e comportamentais dos clientes/paciente; [sendo] encarada também como método de exame capaz de aferir ou dimensionar fenômenos e processos psíquicos” (Alchieri e Cruz, 2009). Nesse contexto figura a Psicometria, definível como a "teoria da medida em Psicologia, que confere medidas aos fenômenos psicológicos, e assim permite que sejam desenvolvidos e construídos testes psicológicos” (Pasquali, 2001). Tal campo de pesquisa quantitativa da Avaliação Psicológica envolve processos como a criação e a adaptação de instrumentos para mensurar fenômenos como a inteligência, personalidade, atitudes, interesses, aptidões, etc (Pasquali, 2009).

A respeito das intervenções psicológicas em pósvenção que empregam instrumentos psicométricos, observa-se as procuram medir características psicológicas como:

- traços de personalidade relacionados ao suicídio (Jobes, Jacoby, Cimbolic, Hustead, 1997);

- níveis de ansiedade e depressão (Paetzold, Silva e Simões, 2021);

- $\quad$ atitude diante do suicídio (Nader, 2012);

- $\quad$ risco de suicídio (Harris et al, 2015; Porte et al, 2020);

- avaliação de possível luto patológico relacionado a dados sociodemográficos (Martins, 2018). 
Em síntese, tais intervenções psicológicas procuram medir riscos de suicídio via atitudes, propensões, para identificar e abordar potenciais suicidas. Isto é, há um foco na prevenção por meio da tentativa de predizer atos quase sempre explicáveis por transtornos mentais ou episódios agudos.

\section{Procedimentos Metodológicos}

A pesquisa apresentada neste artigo é de natureza básica, uma vez que visa criar conceitos para fundamentar teoricamente futuras práticas (Cresswell, 2010); e de caráter tanto exploratório quanto descritivo, uma vez que o presente artigo objetiva analisar questões gerais do tema pouco conhecido da pósvenção e explicar aspectos específicos de tal conceito (Marconi e Lakatos, 2017). A abordagem de pesquisa adotada é quantitativa, tendo os seguintes procedimentos: a) uma revisão integrativa de publicações extraídas de bases de dados científicas; b) a decorrente análise bibliométrica dos achados da revisão integrativa.

No que diz respeito à revisão integrativa, esta foi realizada a partir do modelo de Hopia, Latvala e Liimatainen (2016), que compreende quatro itens:

1. Identificação do problema de pesquisa: “O que a academia tem pesquisado sobre psicometria e pósvenção?”;

2. Definição dos critérios de inclusão: a) estudos publicados sem delimitação de tempo, isto é, em qualquer ano; b) disponibilizados nas bases de dados: Scopus, Web of Science, Pubmed, Base-Search, Science direct, Lilacs, Medline, Psycnet; c) com os seguintes descritores "suicide postvention psychometrics" e "suicídio pósvenção psicometria”; d) apenas artigos publicados em periódicos; e) selecionados a partir da leitura dos títulos, resumos e palavras-chave, conforme o DeCS (Descritores em Ciências da Saúde);

3. Critérios de exclusão para análise dos textos selecionados: a) o primeiro passo foi a retirada dos textos duplicados entre as bases; b) na sequência foi realizada a leitura de 73 abstracts, e destes excluídos 50 textos que contemplavam outros aspectos não relacionados à psicometria aplicada à pósvenção; c) para concluir, foram lidos os textos completos 23 e eleitos os textos relacionados ao objetivo deste estudo (9) (Ver Quadro $1)$;

4. Análise dos resultados: após a leitura na íntegra dos textos eleitos, foram organizados eixos de discussão dos principais resultados (ver seção 4).

Os procedimentos de pesquisa foram realizados conforme o cronograma apresentado no Quadro 1:

Quadro 1 - Etapas do da revisão integrativa Integrativa.

\begin{tabular}{|l|l|}
\hline Etapa & Data \\
\hline Escolha dos termos e filtros de busca de busca & 26 / MAI / 2021 \\
\hline Busca em bases de dados & 02 / JUN / 2021 \\
\hline Seleção das publicações & 08 / JUN / 2021 \\
\hline Análise bibliométrica das publicações selecionadas & 08 / JUN / 2021 \\
\hline
\end{tabular}

Fonte: Autores. 
A seguir, os resultados dos procedimentos realizados, seguidos da discussão dos mesmos e das considerações finais sobre a pesquisa.

\section{Resultados}

\subsection{Publicações e Instrumentos Psicométricos Encontrados}

O Quadro 2 apresenta os primeiros resultados da busca nas bases de dados:

Quadro 2 - Artigos identificados na etapa 2 da revisão a partir de suas bases de dados.

\begin{tabular}{|l|l|}
\hline Base de dados & $\begin{array}{l}\text { Número de } \\
\text { artigos }\end{array}$ \\
\hline Scopus & 32 \\
\hline Science Direct & 25 \\
\hline Psycnet & 13 \\
\hline Pubmed & 2 \\
\hline Base-Search & 1 \\
\hline Medline & 0 \\
\hline Web of Science & 0 \\
\hline Lilaces & 0 \\
\hline
\end{tabular}

Fonte: Autores.

Os 73 artigos resultantes foram filtrados pela leitura dos títulos, resumos e palavras-chave, resultando em 9, expostos no Quadro 3 em ordem cronológica de publicação: 
Quadro 3 - Nove artigos selecionados na revisão integrativa integrativa.

\begin{tabular}{|c|c|}
\hline Título & Instrumento psicométrico \\
\hline "Suicide among physicians: Prevention and postvention" (Simon, 1986) & Sugere a criação de um. \\
\hline $\begin{array}{l}\text { "Assessment of knowledge gains in proactive training for postvention" } \\
\text { (Mackesy-Amiti et. al,1996) }\end{array}$ & Instrumento próprio. Não validado. \\
\hline $\begin{array}{l}\text { "Development of a scale to assess knowledge about suicide postvention using } \\
\text { item response theory" (Nader et al, 2013). }\end{array}$ & $\begin{array}{l}\text { Início do desenvolvimento de um } \\
\text { instrumento próprio. }\end{array}$ \\
\hline $\begin{array}{l}\text { "Fearlessness about Death: The psychometric properties and construct validity } \\
\text { of the revision to the Acquired Capability for Suicide Scale" (Ribeiro et. al, } \\
\text { 2014). }\end{array}$ & $\begin{array}{l}\text { Acquired Capability for Suicide } \\
\text { Scale }\end{array}$ \\
\hline $\begin{array}{l}\text { “A Systematic Assessment of Smartphone Tools for Suicide Prevention” (Larsen, } \\
\text { Nicholas \& Christersen, 2016). }\end{array}$ & $\begin{array}{l}\text { Revisão de questionários empregados } \\
\text { online. }\end{array}$ \\
\hline $\begin{array}{l}\text { "Exploring the association between exposure to suicide and suicide risk among } \\
\text { military service members and veterans" (Hom, Stanley, Gutierrez \& Joiner, } \\
\text { 2017). }\end{array}$ & $\begin{array}{l}\text { Suicidal Behaviors Questionnaire - } \\
\text { Revised (SBQ-R) e o Interpersonal } \\
\text { Needs Questionnaire - Thwarted } \\
\text { Belongingness Subscale (INQ - TB) }\end{array}$ \\
\hline $\begin{array}{l}\text { "Exposure to Suicide in High Schools: Impact on Serious Suicidal } \\
\text { Ideation/Behavior, Depression, Maladaptive Coping Strategies, and Attitudes } \\
\text { toward Help-Seeking” (Gould et al., 2018). }\end{array}$ & $\begin{array}{l}\text { Drug Use Screening Inventory. } \\
\text { The Beck Depression Inventory } \\
\text { (BDI-IA) }\end{array}$ \\
\hline $\begin{array}{l}\text { "Suicide and other sudden death bereavement of immediate family members: An } \\
\text { analysis of grief reactions six-months after death" (Kõlves et al., 2019). }\end{array}$ & $\begin{array}{l}\text { Grief Experience Questionnaire } \\
\text { (GEQ). }\end{array}$ \\
\hline $\begin{array}{l}\text { "The SUPPORT-S Protocol Study: A Postvention Program for Professionals } \\
\text { After Patient or User Suicide” (Leaune et al., 2020). }\end{array}$ & $\begin{array}{l}\text { Differential Emotions Scale IV; } \\
\text { Impact of Event Scale-Revised; } \\
\text { Perceived Social Support Scale for } \\
\text { Professionals; e um questionário não- } \\
\text { validado criado pelos autores. }\end{array}$ \\
\hline
\end{tabular}

Fonte: Autores.

A seguir é descrita a etapa 4 da pesquisa, complementar à revisão integrativa e realizada a partir dos 9 artigos presentes no Quadro 3.

\subsection{Análise bibliométrica das publicações}

O Quadro 4 sintetiza dados bibliométricos das publicações selecionadas, expondo-as em ordem decrescente tomando por base o número de citações: 
Quadro 4 - Bibliometria dos artigos selecionados.

\begin{tabular}{|c|c|c|c|c|c|}
\hline Título do artigo & Citações & Periódico & País & Área de publicação & $\begin{array}{l}\text { Impact } \\
\text { Factor }\end{array}$ \\
\hline $\begin{array}{l}\text { "Fearlessness about Death: The psychometric } \\
\text { properties and construct validity of the revision to the } \\
\text { Acquired Capability for Suicide Scale" (Ribeiro et. } \\
\text { al, 2014). }\end{array}$ & 237 & $\begin{array}{l}\text { Journal of } \\
\text { Psychological } \\
\text { Assessment }\end{array}$ & EUA & Psicologia & 2.825 \\
\hline $\begin{array}{l}\text { "Exploring the association between exposure to } \\
\text { suicide and suicide risk among military service } \\
\text { members and veterans" (Hom, Stanley, Gutierrez \& } \\
\text { Joiner, 2017). }\end{array}$ & 27 & $\begin{array}{l}\text { Journal of affective } \\
\text { disorders }\end{array}$ & EUA & Psiquiatria & 3.892 \\
\hline $\begin{array}{l}\text { "Suicide among physicians: Prevention and } \\
\text { postvention" (Simon, 1986) }\end{array}$ & 22 & $\begin{array}{l}\text { The Journal of Crisis } \\
\text { Intervention and } \\
\text { Suicide Prevention }\end{array}$ & Austrália & Medicina & 1.482 \\
\hline $\begin{array}{l}\text { "Exposure to Suicide in High Schools: Impact on } \\
\text { Serious Suicidal Ideation/Behavior, Depression, } \\
\text { Maladaptive Coping Strategies, and Attitudes toward } \\
\text { Help-Seeking” (Gould et al., 2018). }\end{array}$ & 10 & $\begin{array}{l}\text { International Journal } \\
\text { of Environmental } \\
\text { Research and Public } \\
\text { Health }\end{array}$ & Suiça & Multidisciplinar & 2.849 \\
\hline $\begin{array}{l}\text { "Suicide and other sudden death bereavement of } \\
\text { immediate family members: An analysis of grief } \\
\text { reactions six-months after death" (Kõlves et al., } \\
\text { 2019). }\end{array}$ & 5 & $\begin{array}{l}\text { Journal of affective } \\
\text { disorders }\end{array}$ & EUA & Psiquiatria & 3.892 \\
\hline
\end{tabular}

Fonte: Autores.

No Quadro 4, há duas colunas que expressam indicadores bibliométricos: "Citações", cuja fonte é a base de dados online Semantic Scholar, e expressa o número de citações do artigo realizadas por outros autores; e o "Impact Factor", indicador bibliométrico que quantifica a relevância, em citações, do periódico.

\section{Discussão dos Resultados}

\subsection{Crítica do conceito de pósvenção}

Constatou-se, pela leitura dos artigos selecionados na revisão integrativa, que o termo "pósvenção" comumente é substituído por “intervenção posterior (ao suicídio)" e demais sinônimos, como "tratamento pós-suicídio”, “cuidados subsequentes" e afins. Presume-se que publicações que abordam práticas classificáveis como pósvenção podem até mesmo nunca citá-la nominalmente. 
A prática da pósvenção sempre surge atrelada à prevenção. A ponto de ser prudente questionar se aquela não consiste apenas em uma modalidade diferenciada desta. Em outras palavras: será a pósvenção algo de fato novo, ou apenas uma especialização da prevenção? A leitura das publicações selecionadas sugere a segunda alternativa como a mais provável. Isto é, pósvenção como, em parte, uma especialização da prevenção em cenários onde uma crise relacionada a suicídio já está intalada e portanto o trabalho de prevenção é de caráter emergencial. No que a pósvenção se diferencia da prevenção, propriamente: envolve contato direto e delicado com os enlutados num contexto de crise intensa.

A pósvenção confirma-se como multidisciplinar, dada a variedade de áreas dos artigos: Medicina (Simon, 1986), Serviço Social (Leaune et al, 2020); Educação escolar (Gould et al, 2018). Os demais artigos tinham público-alvo geral. O que aponta para a necessidade do contato com os enlutados ser feito por um time multidisciplinar.

\subsection{Psicometria da pósvenção}

Os artigos selecionados possuem uma ênfase instrumental e programática. Um dos artigos consiste na criação de programa de pósvenção (Leaune et al, 2020) e quatro consistem no desenvolvimento ou melhoria de instrumentos psicométricos (Simon, 1986; Ribeiro et al, 2014; Gould et al, 2018; Nader et al, 2013 e Mackesy-Amiti, 2010). Tal fenômeno pode ser explicado pela escassez de instrumentos psicométricos feitos para pósvenção, objeto de estudo recente e pouco explorado.

As intervenções concentram-se em investigar como os enlutados reagem ao suicídio, isto é, como fica a saúde mental dos enlutados para fins de monitoramento de risco. Os construtos psicológicos investigados psicometricamente, conforme os artigos selecionado na revisão, foram: medo da morte (Ribeiro et al, 2014); comorbidades a ideação suicida (Gould, 2018); conhecimento a respeito de como intervir em pósvenção (Nader et al, 2013) e aprendizado sobre pósvenção via treinamentos (Mackesy-Amiti, Fendrich, Libby e Goldenberg 2010). A direção dos estudos se concentra em avaliar o risco dos enlutados em termos de saúde mental, bem como a capacidade de ser ele mesmo um agente de pósvenção.

Pode-se interpretar padrões nos nove artigos selecionados na revisão integrativa:

a) Avaliação de fatores de riscos pré-existentes, tais como tendências à depressão e ao abuso de substâncias (Gould et al., 2018);

b) Aquisição de ideações suicidas ocorrida durante o luto (Ribeiro et al, 2014);

c) Potenciais de saúde presentes no ambiente do enlutado. No nível individual, a capacidade emocional de lidar com stress (Kõlves et al., 2019) e a aptidão para elaborar luto (Hom, Stanley, Gutierrez \& Joiner, 2017), e no nível coletivo, o senso de pertencimento social e suporte familiar e comunitário recebido (Leaune et al, 2020).

Destaca-se dois usos destoantes do conjunto: a) um dos artigos revisa ferramentas de mobile phone para pósvenção (Larsen, Nicholas e Christensen, 2016), o que também aponta para a busca da proatividade na própria comunidade de enlutados para mútuo apoio e superação da crise; b) outro artigo apresenta testes psicométricos para checkups regulares de saúde emocional de médicos para fins de seleção e promoção em hospitais (Simon, 1986).

\subsection{Países de origem das pesquisas}

Todas as publicações resultantes da revisão integrativa são provenientes de países anglo-saxões (EUA, Grã-Bretanha, Austrália e Suíça). Diante disso foram levantadas as seguintes hipóteses: a) nesses países ocorrem mais casos de suicídio que despertam maior interesse por pósvenção; b) em tais países há melhor infraestrutura para avaliação psicológica em instituições; c) tais países possuem facilidades culturais para a aplicação da psicometria em pósvenção. A hipótese "a" foi descartada, dadas as estatísticas epidemológicas do suicídio em países como o Brasil (Ministério da Saúde, 2019). Entende-se que a hipótese "b" e a "c" demandam estudos posteriores para melhor avaliação. 
Não foram encontradas publicações brasileiras. Como visto na seção 1 deste artigo, a produção acadêmica nacional sobre pósvenção parece começar na segunda metade da década de 2010.

\subsection{Análise bibliométrica das publicações}

A partir dos indicadores bibliométricos (ver Quadro 4), pode-se observar que as publicações que combinam mais citações e periódicos de maior impacto são aquelas sobre criação de instrumentos psicométricos para uso geral (Ribeiro et al, 2014) e revisão de instrumentos (Larsen, Nicholas e Christensen, 2016). Enquanto que as publicações com valores menores possuem o termo "postvention" no título, sendo portanto específicas da área. Estas são as que se propõem a criar uma escala de avaliação de conhecimento (Nader et al, 2013) e um protocolo de intervenção (Leaune et al, 2020).

A análise bibliométrica dos resultados da revisão permite inferir, portanto, que as psquisas mais instrumentais e gerais despertam maior interesse da comunidade. Isso pode ser explicado pelo histórico recente e ainda escasso recente de pesquisa em pósvenção. Contudo, como a revisão integrativa identificou apenas 9 publicações. Por isso talvez tal análise falhe em seu poder preditivo por razão amostral.

\subsection{Recenticidade das pesquisas}

Por fim, observa-se como as publicações são, na maioria, da década de 2010 em diante, apesar do termo "pósvenção" datar de 1968 (New York State Psychiatric Institute, 2020). A essa regra tem-se duas exceções: Simon (1986) e Mackesy-Amiti et al (1996). O que sugere que a problemática da pósvenção assistiu o interesse da comunidade de pesquisadores ser aumentado consideravelmente há poucos anos. Nota-se ainda que as publicações de 2017 em diante fazem uso de instrumentos psicométricos validados, enquanto as mais antigas apenas sugerem ou iniciam o desenvolvimento de algum instrumento, o que possivelmente evidencia o progresso do conhecimento psicométrico da pósvenção ao longo dos anos.

Os programas de pósvenção ainda são raros e declarações sobre a falta de rigor e evidências científicas na área são comuns entre pesquisadores (Ramchand et al, 2015). A delicada problemática do suicídio costuma ser tratada, aparentemente, com intervenções pautadas mais por empirismo do que por ciência. Os programas existentes, criados para instituições escolas e militares, fazem uso de protocolos baseados principalmente em pesquisa qualitativa, destacando-se os grupos focais e as entrevistas individuais como procedimentos de pesquisa. Os pesquisadores que descrevem esses programas apontam a falta de procedimentos quantitativos e a necessidade de pesquisas em larga escala que façam uso integrativo dos mesmos.

\section{Considerações Finais}

Os autores deste artigo entendem que o objetivo da pesquisa foi atendido, uma vez que foram levantadas formas como a Psicometria vem sendo usada para fundamentar intervenções quantitativas em pósvenção. Observou-se como a Psicometria é aplicada para avaliar o estado psicológico dos enlutados, em busca de sinais de risco, bem como com o potencial de saúde e resiliência dos mesmos, e até de colaborarem entre si para formação de redes de apoio.

No que diz respeito aos procedimentos empregados neste estudo, a revisão integrativa sempre apresenta limitações de escopo e abrangência. Dado o histórico recente da pósvenção talvez artigos de menor repercussão, oriundos de congressos, por exemplo, devessem ter sido incluídos nos resultados. Talvez a pósvenção como objeto de estudo ainda seja recente demais para ser estudada por revisões amplas, demandando estudos de caso e pesquisas qualitativas em profundidade.

Constam como sugestões dos autores para estudos futuros: 1) a pósvenção no Brasil, a partir dos desafios de como nossa cultura pensa e sente o suicídio; 2) adaptação às peculiaridades culturais e regionais brasileiras de programas e instrumentos de pósvenção desenvolvidos em outros países; 3) o desenvolvimento e validação de instrumentos psicométricos para embasar 
programas de pósvenção no Brasil; 4) programas de pósvenção segmentados por profissões e ambientes institucionais. Ex.: militares, profissionais de saúde hospitalar, comunidade escolar, etc.

\section{Referências}

Alchieri, J. C. \& Cruz, R. M. (2009). Avaliação psicológica: conceito, métodos, medidas e instrumentos. (2a ed.), Casa do Psicólogo.

American Psychiatric Association. (2014). Manual Diagnóstico e Estatístico de Transtornos Mentais: DSM-5. Artmed.

Andriessen, K., Krysinska, K., \& Grad, O. T. (2017). Postvention in Action. Hogrefe.

AQPS (2008). Association Québécoise de Prévention du Suicide. https://www.aqps.info/

Betti, E. K. (2020). Prevenção e pósvenção ao suicídio: cuidados que os serviços de saúde oferecem aos enlutados por suicídio. Trabalho de Conclusão de Curso em Psicologia. UNISC.

Cresswell, J. W. (2010). Projeto de pesquisa: métodos qualitativo, quantitativo e misto. Artmed.

Conselho Regional de Psicologia - 12. (2020). Orientações para a atuação profissional frente a situações de suicídio de automutilação. http://conselho.saude.gov.br/images/CRPDF-Orientacoes_atuacao_profissional.pdf

Durkheim, E.(1982). O Suicídio - Um Estudo Sociológico. Zahar Editores.

Erbacher, J. B. \& Singer, S. P. (2015). Suicide in Schools. A Practitioner's Guide to Multi-level Prevention, Assessment, Intervention, and Postvention. Routledge.

Fukumitsu, K. O. (2014). O psicoterapeuta diante do comportamento suicida. Revista de Psicologia da USP. 25 (3), $270-275$.

Fukumitsu, K. O (2018). Posvenção: uma intervenção dolorida, porém necessária. https://jornal.usp.br/artigos/posvencao-uma-intervencao-dolorida-poremnecessaria/

Gould, M.S., Lake A.M., Kleinman M., Galfalvy, H., Chowdhury, S. \& Madnick A.(2018). Exposure to Suicide in High Schools: Impact on Serious Suicidal Ideation/Behavior, Depression, Maladaptive Coping Strategies, and Attitudes toward Help-Seeking. Int J Environ Res Public Health, 15 (3):455.

Hopia, H., Latvala, E. \& Liimatainen, L. (2016). Reviewing the methodology of an integrative review. Scand J Caring Sci. 30(4), 662-9. DOI: https://doi.org/10.1111/scs.12327

Ibrahim, N., Che Din, N., Amit, N., Ghazali, SE, \& Mohd Safien, A. (2019). Development and validation of Yatt Suicide Attitude Scale (YSAS) in Malaysia. PloS one, 14 (2), e0209971.

Kreuz, G. \& Antoniassi, R. P. N. (2020). Grupo de apoio para sobreviventes do suicídio. Psicologia em Estudo, 25, e42427. https://doi.org/10.4025/psicolestud.v25i0.42427

Guimarães, SE. R., Santos, M. C. \& Lima, M. (2020). Políticas públicas de saúde na prevenção e posvenção do comportamento suicida: uma revisão de literatura. Anais do I Colóquio de Pesquisa em Psicologia. FG. UNFG.

Harris, K. M., Syu, J., Lello, O D., Chew, Y. L. E., Wilcox, C. H., Ho, R. H. M. (2015). The ABC's of Suicide Risk Assessment: Applying a Tripartite Approach to Individual Evaluations. PLOS ONE 10(7): e0133223. https://doi.org/10.1371/journal.pone.0133223

Hom, M. L., Stanley I. H., Gutierrez, P. M. \& Joiner, T. E. (2017). Exploring the association between exposure to suicide and suicide risk among military service members and veterans. Journal of Affect Disorders. 207:327-335. doi: 10.1016/j.jad.2016.09.043. Epub 2016 Sep 28. PMID: 27743535.

Jobes, D. A., Jacoby, A. M., Cimbolic, P., \& Hustead, L. A. T. (1997). Assessment and treatment of suicidal clients in a university counseling center. Journal of Counseling Psychology, 44(4), 368-377. https://doi.org/10.1037/0022-0167.44.4.368

Jordan, J. R. (2017). Postvention is prevention - The case for suicide postvention. Death studies, 41(10), 614-621. https://doi.org/10.1080/07481187.2017.1335544

Kõlves, K., Zhao, Q., Ross, V., Hawgood, J., Spence, S.H. \& de Leo, D (2019). Suicide and other sudden death bereavement of immediate family members: An analysis of grief reactions six-months after death. J Affect Disord. 243:96-102. 10.1016/j.jad.2018.09.018. Epub 2018 Sep 12. PMID: 30241027.

Larsen, M.E., Nicholas, J. \& Christensen, H. (2016). A Systematic Assessment of Smartphone Tools for Suicide Prevention. PLOS One. 11(4):e0152285. 10.1371/journal.pone.0152285.

Leaune, E., Cuvillier, B., Vieux, M., Pacaut-Troncin, M., Chalancon, B., Perez, A.F., Haesebaert, J., Chauliac, N., Poulet, E. \& Durif-Bruckert, C. (2020). The SUPPORT-S Protocol Study: A Postvention Program for Professionals After Patient or User Suicide. Front Psychol. 11.805. 10.3389/fpsyg.2020.00805.

Presidência da República (1962). Lei no 4.119, de 27 de agosto de 1962. http://www.planalto.gov.br/ccivil_03/leis/1950-1969/14119.htm

Marconi, M. A. \& Lakatos, E. M. (2017). Metodologia do trabalho científico. (8a ed.), Editora Saraiva.

Mackesy-Amiti, M.E., Fendrich, M., Libby, S., Goldenberg, D. \& Grossman J. (1996). Assessment of knowledge gains in proactive training for postvention. Suicide Life Threat Behav. 26(2):161-74. PMID: 8840420. 
Martins, A. C. S. (2018). Famílias Enlutadas Face ao Suicídio - Distrito de Coimbra de 2013 a 2016. https://eg.uc.pt/handle/10316/82026

Michel, L. H. F. \& Freitas, J. L. (2019). A clínica do luto e seus critérios diagnósticos: possíveis contribuições de Tatossian. Psicologia USP. 30.

Müller, S. A., Pereira, G. S. \& Zanon, R. B. (2017). Estratégias de prevenção e pósvenção do suicídio. Revista de Psicologia da IMED, 9 (2), 6-23

New York State Psychiatric Institute. (2020). Suicide Contagion Among Adolescents. https://cdpsdocs.state.co.us/safeschools/Resources/Virtual_Train ings/SSS_2020/Gould_Contagion_CO_Safe_Schools_Oct_2020.pdf

Nader, I. (2012). Application of novel statistical methods in suicide research. Dissertation, University of Vienna. Fakultät für Psychologie.

Nader I., Niederkrotenthaler, T., Schild, A.H., Koller, I., Tran, U.S., Kapusta, N.D., Sonneck, G. \& Voracek, M. (2013). Development of a scale to assess knowledge about suicide postvention using item response theory. Suicide Life Threat Behav. 43(2):174-84.

Nogueira, A.V., Ostrowski, E.C. \& Knapik, J. (2019). Suicídio no Brasil: o papel da posvenção como intervenção psicológica - Revisão de Literatura. Trabalho de Conclusão de Curso em Psicologia.Universidade Positivo.

Nunes, E. D. (1998). O Suicídio - reavaliando um clássico da literatura sociológica do século XIX. Cadernos de Saúde Pública. 14(1):7-34.

Olinda, K. R. S., Santos, R. F. \& Marques, T. H. C. (2020). Fatores associados ao comportamento suicida em hospital psiquiátrico: estudo transversal. Revista Eletrônica Acervo Saúde, 12 (6), e3050.

Pasquali, L. (2001). Técnicas de Exame Psicológico - TEP. Vol. I: Fundamentos das Técnicas de Exame Psicológico. Casa do Psicológo, São Paulo.

Pasquali, L. (2009). Psicometria. Revista da Escola de Enfermagem da USP, 43.

Paetzold, M. G., Silva, L. L. \& Simões, M R. (2021). Métodos de rastreamento da ansiedade e depressão em estudantes universitários: revisão integrativa. Saúde Coletiva, 11(60), https://doi.org/10.36489/saudecoletiva.2021v11i60p4686-4695

Barbosa, E. D. (2017). Suicídio: um olhar para os sobreviventes. https://app.uff.br/riuff/handle/1/6463

Wertheimer, S., Barnes-Proby, D.,, \& Ramchand, R. R.(2015). Suicide Postvention in the Department of Defense: Evidence, Policies and Procedures, and Perspectives of Loss Survivors. Rand Foundation.

Ribeiro, J. D., Witte, T. K., Van Orden, K. A., Selby, E. A., Gordon, K. H., Bender, T. W. \& Joiner, T. E. (2014). Fearlessness about death: the psychometric properties and construct validity of the revision to the acquired capability for suicide scale. Psychol Assess., 26(1):115-26. 10.1037/a0034858.

Santos, L. C. (2019) As Limitações e Potencialidades na Prevenção, Intervenção e Pósvenção do Suicídio na Guarda Nacional Republicana. http://comum.rcaap.pt/handle/10400.26/30103

Santos, R. S., Albuquerque, M. C., Brêda, M. Z., Bastos, M. L. A. , Silva, V. M.S. \& Tavares, N. V. S. (2017). A atuação do enfermeiro com a pessoa em situação de suicídio: análise reflexiva. Revista de Enfermagem da UPPE, 11(2).

Secretaria de Justiça e Cidadania do Distrito Federal (2019). Protocolo de prevenção e atenção ao suicídio de adolescentes no sistema socioeducativo. http://www.sejus.df.gov.br/wp-conteudo/uploads/2019/10/Protocolo-Preven\%C3\%A7\%C3\%A3o-ao-Suic\%C3\%ADdio-copiar-mesclado-p\%C3\%A1ginas-1911-1620-27.pdf

Silva, L G. B. \& Marinho, C. A. S. (2017). Suicídio: Aspectos reacionais e o processo de elaboração do luto na família. https://www.ippesbrasil.com.br/wpcontent/uploads/2018/06/A1137.pdf

Simon, W. (1986). Suicide among physicians: Prevention and postvention. Crisis: The Journal of Crisis Intervention and Suicide Prevention, 7(1), 1-13.

California State University (2006). Suicid Postvention. Suggested procedures during the aftermath os a student suicide. https://www.yumpu.com/en/document/read/22015465/suicide-postvention-california-state-university-sacramento

Vita Alere, 2018. O que é posvenção? https://vitaalere.com.br/sobre-o-suicidio/posvencao/o-que-e-posvencao/

Wainwright, V., Cordingley, L., Chew-Graham, C. A., Kapur, N., Shaw, J., Smith, S., \& McDonnell, S. (2020). Experiences of support from primary care and perceived needs of parents bereaved by suicide: a qualitative study. British journal of general practice,70(691), e102-e110. : https://doi.org/10.3399/bjgp20X707849

World Health Organization, Age-standardized suicide rates. https://www.who.int/data/gho/data/themes/mental-health 